\title{
Obstacle Avoidance Cell Discovery using mm-waves Directive Antennas in 5G Networks
}

\author{
Antonio Capone*, Ilario Filippini*, Vincenzo Sciancalepore ${ }^{\dagger \ddagger *}$, Denny Tremolada* \\ * DEIB - Politecnico di Milano $†$ IMDEA Networks Institute $\ddagger$ Universidad Carlos III de Madrid
}

\begin{abstract}
With the advent of next-generation mobile devices, wireless networks must be upgraded to fill the gap between huge user data demands and scarce channel capacity. Mm-waves technologies appear as the key-enabler for the future $5 \mathrm{G}$ networks design, exhibiting large bandwidth availability and high data rate. As counterpart, the small wave-length incurs in a harsh signal propagation that limits the transmission range. To overcome this limitation, array of antennas with a relatively high number of small elements are used to exploit beamforming techniques that greatly increase antenna directionality both at base station and user terminal. These very narrow beams are used during data transfer and tracking techniques dynamically adapt the direction according to terminal mobility. During cell discovery when initial synchronization must be acquired, however, directionality can delay the process since the best direction to point the beam is unknown. All space must be scanned using the tradeoff between beam width and transmission range.

Some support to speed up the cell search process can come from the new architectures for 5G currently being investigated, where conventional wireless network and mm-waves technologies coexist. In these architecture a functional split between C-plane and U-plane allows to guarantee the continuous availability of a signaling channel through conventional wireless technologies with the opportunity to convey context information from users to network.

In this paper, we investigate the use of position information provided by user terminals in order to improve the performance of the cell search process. We analyze mm-wave propagation environment and show how it is possible to take into account of position inaccuracy and reflected rays in presence of obstacles.
\end{abstract}

\section{INTRODUCTION}

The 5th generation $(5 \mathrm{G})$ of wireless networks is currently gaining the momentum in the research community. Several research activities are focused on new challenges while the smartphone and tablet revolution is strongly affecting the wireless network capacity. Providing 1000 times higher traffic volumes and guaranteeing high peak rates is no longer an option: advanced solutions must be considered to deal with network resource scarcity. The compelling limitation of available frequency band is drawing the attention of academic and industry partners which exert to use frequencies above the conventional $5 \mathrm{GHz}$. In this context, the advantage of exploiting millimiter waves (mm-waves) communication at $60 \mathrm{GHz}$ is two-fold: $i$ ) unlicensed spectrum band can be smartly managed and $i$ ) wide bandwidths (up to $1 \mathrm{GHz}$ ) may lead to high data rates performance during communications [1].

Although mm-waves channels are attractive and represent the most promising solution for future network design, they suffers from the major drawback due to the coarse propagation at very-high frequencies. Refraction and reflection effects are adversely hampered by the presence of obstacles along the communication path. Therefore, modeling the propagation channel under those conditions becomes very challenging due to huge number of parameters to be tuned [2]. Fortunately, while the very small wave-length negatively affects the channel attenuation, this readily allows to use much more antenna elements in very limited areas (suitable for mobile devices). This straightforwardly enables the beamforming technique to build a quasi-errorless channel between two physical transmitters in light of sight. On the one hand, the directive transmissions consolidate the effective power over small angle and dramatically extends the coverage range up to few hundred meters. On the other hand, transmitters and receivers must beam out each others beforehand to establish a proper connection by scanning over all possible directions due to the confined viewing angle [3]. The overdue time during the synchronization process between mm-waves small cells and User Equipments (UEs ${ }^{1}$ ) may delay or brutally causes a service disruption in the mm-waves access network [4].

Interestingly, designed system architectures rely on heterogeneous networks where legacy communication technologies have not been yet surmounted. Continuous connectivity service is always guaranteed while switching on future mm-waves access network to boost the network capacity only when available. For this propose, advanced system architectures are currently under the evaluation of different research projects, such as FP7-ICT EU MiwaveS project [5] or FP7-ICT EU MiWeba project [6]. Additionally, the latter assumes a function split between the user (U-) plane and control (C-) plane when signaling transmissions could be compromised by the unexpected lack of connectivity [7]. Thus, signaling messages are exchanged through conventional communication channels leaving the full management of data connections under the control of mm-wave network functions, as envisaged in Fig 1. The availability of a unfailing signaling layer benefits the network functions related to mobility management and service access. For instance, context information may be available at the mm-waves small cells, which may successfully retrieve information about user positions to minimize the delay for the cell discovery process (also defined as cell search process).

In this paper, we rely on the context-based solution to asses the cell discovery performance when newcoming users join the network. Specifically, an external localization system is involved (e.g., GPS) and user position information are facilitated through the conventional network. However, the compound effect of signal obstruction, due to the presence

\footnotetext{
${ }^{1}$ Both terms UE and users are used interchangeably throughout the paper.
} 


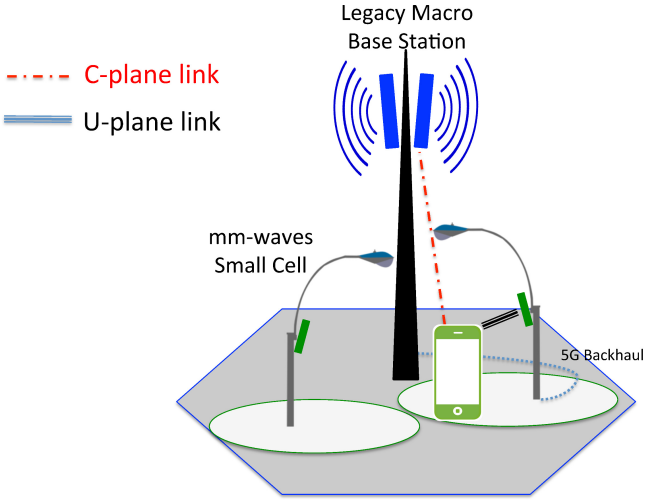

Fig. 1: Heterogenous system architecture with a C-/U-plane function split.

of obstacles in the area network, with the inaccuracy of the location information may seriously abate the cell discovery performance. We extend the fast cell discovery algorithm presented in [4] by means of a traditional training and learning approach, and provide full details on how our algorithm properly handles the $\mathrm{mm}$-waves propagation issues.

The paper is organized as follows. In Section II we review the state of the art, shedding the light on our novel contributions. In Section III, the cell discovery issues are commented, showing how our solution speed up the discovery process. Section IV presents our simulation results providing insightful comments, and in Section V we conclude the paper.

\section{RELATED WORK}

The current development of WiGig standard specification, namely 802.11ad, brings the mm-wave potential into Wireless LAN environments, where similar issues are addressed. Specifically, the beamforming training is performed at frame level, where a sector exploration is carried out by the antenna. In [8] a novel MAC protocol is proposed to switch between the best antenna direction, detected during the initial phase, and the relay station direction when channel degradation occurs. Also in [9], authors propose an algorithm to optimally select a beam-width while guaranteeing that a subset of users are reached and connected. This results in a high channel occupation and very low number of collisions during transmissions.

Similar problems have been already investigated in the adhoc wireless network design, where directional antennas have been used to provide C-plane network functions. Although improving the spatial reuse and extending the link range, they aggravate deafness and hidden terminal problems increasing the complexity of the neighbour discovering phase. Therefore, they typically adopted solutions either consider the presence a control channel established via omnidirectional interfaces to orchestrate the beamforming or assume an initial training phase where the correct direction to point each neighbour is found through a complete scan of the space [10], [11], [12].

Finally, the cell discovery problem in mm-wave cellular networks has been already considered in [13], [3], [4]. While in [3], authors prove a mismatch between the area where the network is discoverable and the area where the mm-wave service is available, in [13] the cell discovery procedure is addressed from a physical perspective and a solution is proposed. Lastly, [4] presents an interesting cell search algorithm which optimally exploits the context-based information.

With respect to previous literature, the novel contributions we provide in this paper can be summarized as follows: $i$ ) we propose an enhanced version of a cell discovery procedure which exploits ray reflections to avoid channel obstructions, ii) we introduce a learning approach to store successful beamforming configurations which are used for future user detections, iii) we validate our approach showing outstanding results against a trivial greedy search approach.

\section{FASt Cell Discovery}

Synchronisation and user acquisition involve many challenges when mm-waves communication is enabled. In particular, ultra-high frequency communications require an additional discovery phase when directive antennas are deployed. Upon a new user gets the ultra-capacity coverage provided by the availability of a mm-waves technology, the legacy network layer conveys the synchronization request to the mm-waves small cell. Mw-waves small cell starts the cell discovery process to beam out the newcomer and correctly establish the connection. When the UE is connected, tracking and tracing procedures enable the mm-waves small cell to start the association phase with the user. This phase is mostly affected by the time delay within the UE is discovered. Assuming that users are provided with omnidirectional antennas, as the limited space on the UE side, we cast the cell discovery problem into a time minimization problem subject to the fact that $\mathrm{mm}$-waves small cell does find the appropriate beamforming configuration to reach the user. The rationale behind is that a large delay in the discovery phase might cause a service interruption and provides a deplorable quality of service.

However, the cell discover phase can be significantly improved when context information are available on the conventional cellular network while C-/U-plane function split is in place. For instance, context information may include neighbouring beamforming configurations which aids the intercell interference coordination [14] or even include UE information, such as UE spatial distribution or UE positions provided by an external positioning system. Indeed, the context knowledge successfully helps the mm-waves small cell to properly take decisions during the cell discovery phase, which may be greatly reduced. As counterpart, unavoidable uncertainty in the user position may severely hurt the cell discovery performance. Furthermore, even full position knowledge at the mm-waves small cell side might be not sufficient to directly beam the user. When physical objects act as obstacles within the mm-waves transmission path, the transmission rate could be drastically dropped and user could be not reached at all. In order to address all the above-mentioned issues, we need to devise a smart cell search algorithm without incurring in intractable system complexity. In the following we rely on the approach presented in [4] and we detail an extended version which efficiently handles $i$ ) obstacles in the communication path and ii) uncertainty user position issues. 


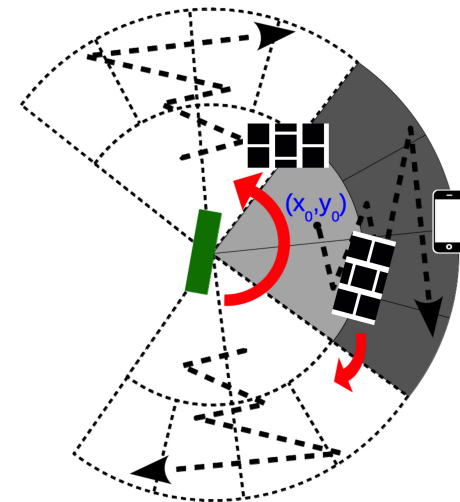

Fig. 2: Example of Enhanced Discovery Procedure (EDP). Estimated user location is $\left(x_{0}, y_{0}\right)$ and two obstacles are placed in the area.

\section{A. Baseline Scenario}

The adoption of a small wave-length critically exacerbates the cellular channel conditions exhibiting a strong pathloss effect due to the very-high frequencies involved. Directive antenna transmissions ease the bad channel problem focusing the antenna gain on a limited view angle. Assuming a 2D exploration, the cell discovery procedure could properly set the antenna view angle, namely beam-width $\phi$, to speed up the cell search process. When the beam-width is pretty wide, coverage range is poor and mm-waves small cell is able to cover only center-users. Conversely, a narrow beam-width allows to reach faraway users but requiring high pointing accuracy. Once the beam-width is fixed, a spatial exploration (e.g., choose direction $d$ ) is required to hook the user synchronisation signal.

Considering a obstacle-free environment, where mm-waves small cell has a perfect knowledge about user position, the spatial exploration is trivially reduced to one single attempt, i.e., the mm-waves small cell computes the optimal beamforming configuration pair $(\phi, d)$ and beam directly the user. Differently, inaccuracy of context information may lead to a wrong beamforming parameters calculation and unsuccessful user recognition. This compels the mm-waves small cell to start a pure search over different direction or even change the beam-width $\phi$ is no user has been found. Based on that, a simple greedy algorithm is proposed in [4]. Starting from the largest beam-width $\phi$ the so-called Discovery Greedy Search (DGS) performs an exhaustive sequential search over all possible directions. If no user is detected, the beam-width $\phi$ is reduced and the spatial exploration starts again. While this guarantees that all possible beamforming configuration pairs are correctly evaluated, the entire process may suffer from significant delays. Therefore, choosing a beam-width value becomes a crucial decision: the thinner the beam-width, the more directions must be explored to find out the user. Additionally, a larger beam-width is more robust to uncertain position information exchanged through the context channel.

\section{B. Obstacles and reflections effects}

Real environments impose that some object can lie between mm-waves transmitters placed in different locations, causing a severe signal attenuation. Those obstructions could be caused by fixed obstacles (tree, buildings, and so on) or by dynamic objects (human body, vehicles, train and so on). While obstacle motion can be modelled as fading effects and can be addressed by physical communication layer solutions, fixed obstacles might drop the signal and must be avoided. Since we consider all objects in the field as opaque obstacles, ray reflections due to the ground or to other obstacle surfaces cannot be negligible. Therefore, when user is not reachable due to a lack of direct transmission path, the cell search algorithm exploits the ray reflection effect to provide the user with a minimum level of power. In our study, we model the power due to reflection as follows:

$$
P_{r}=20 \log _{10}\left(\frac{\lambda}{4 \pi d}\right)-A_{0} d+R+F
$$

where

$$
\begin{aligned}
& R=20 \log _{10}\left(\frac{\sin \theta-\sqrt{B}}{\sin \theta+\sqrt{B}}\right), B=\epsilon-\cos ^{2} \theta \\
& F=\frac{-80}{\ln (10)}\left(\frac{\pi \sin \theta \sigma}{\lambda}\right)^{2} .
\end{aligned}
$$

We consider $\epsilon$ and $\sigma$ as the roughness and reflection coefficients of the material, while $\theta$ as the angle of reflection. We refer the reader to [15] for further details. Ideally, whenever the network is perfectly aware about user locations and fixed obstacles positions in the surrounded area, the cell discovery procedure is able to promptly calculate beamforming parameters (beam-width and pointing direction), avoiding successfully the obstacles. In practice, semi-static obstacles in conjunction with partial UE position information, might notably delay the cell discovery process, even when advanced algorithm are implemented.

\section{Enhanced Discovery Procedure}

We proposed an enhanced version of the algorithm presented in [4], namely Enhanced Discovery Procedure, using a learning approach while running. The algorithm takes into account the possibility of obstacle avoidance using reflected rays while exploiting the context information to reduce the user discovery time.

In the previous scheme, when a user position is retrieved through the context channel, the cell search algorithm computes the optimal beamforming parameters to spot the user. If low accuracy is provided and user is not found, the algorithm looks around the pre-selected direction. Most likely the real user position is very close to the wrong announced place. Thus, EDP sequentially explores $i$ ) adjacent directions (following clockwise and counter-clockwise directions) keeping fixed the beam-width, and $i$ ) farther positions by reducing the beamwidth, as envisaged in Fig 2. However, user can be unreachable in that particular area portion (namely, discovery sector) because of an obstacle obstruction along the exploration path, and thus, EDP needs to move to the next sector to explore ray reflected paths.

To overcome that, we modified the EDP scheme introducing a memory-store feature which learns about successful choices. Whenever the user is discovered, we store the correct beamforming configuration in a fingerprint map, which keeps 
track about user positions and beamforming parameters. This approach takes advantage of previous explorations, speeding up the process when a new user comes close to a known location. However, the user discovery failure could be even caused by an inaccurate user position information and thus, we need to tune properly the learning scheme to use carefully the stored information. We introduce a memory-range $m$ which represents the maximum error deviation with respect to the store position. When a user announces its position within $m$ meters from a stored positions, the corresponding beamforming configuration is used to hook the user synchronization signal. If multiple stored positions are considered, EDP uses the configuration corresponding to the closest location. A large memory-range $m$ will guide EDP to beam several incoming users relying on stored information, but it may lead to wrong beamforming parameters and thus, to a long discovery time. Conversely, a short memory-range will be more accurate but needs much more successful discoveries to work optimally.

\section{Performance eVAluation}

We carried out an exhaustive simulation campaign to evaluate our enhanced approach (EDP) when learning feature is applied. For the sake of completeness, we benchmark EDP against the greedy search algorithm (DGS), presented in [4]. Numerical results are obtained through a MATLAB simulator, developed ad-hoc. Each presented value is averaged over 100 simulation instances. In the following we show the implementation details. All antenna gains are modelled as a Gaussian main lobe profile and log-distance path loss model is implemented, where the pathloss reference value is equal to 82.02dB with a reference distance equal to 5 meters. Reflected ray follows the propagation model shown in Eq. (1), where $\sigma=0.2 \mathrm{~mm}$ and $\epsilon=4+0.2 j$. Full details are provided in [15]. We assume a sufficient signal level for user acquisition directly derived from the empirical measurements presented in [13], where a Signal-to-Noise-Ratio (SNR) must be greater than $10 \mathrm{~dB}$. Cell discovery procedure explores beamforming configurations only over a $2 \mathrm{D}$ plane. The smallest selectable beam-width $\phi$ is 0.0157 rad which leads to a spatial exploration of 360 non-overlapping directions $d$. Larger beam width are obtained by reducing proportionally the number of directions to $180,120,90,72,60,48,24,12,8,6,4,3,2$. Baseline scenario presents a deployment area of $450 \times 350$ meters where $1 \mathrm{~mm}$-wave small cell is placed in the middle and 250 users randomly dropped in the area.

\section{A. Obstacle-free setup}

In the first set of results we want to study how learning approach impacts on the cell discovery time. Please note that we evaluate system performance in terms of number of beamforming parameter switches (or number of attempts to successfully hook the user synchronization signal), which is straightforwardly related to the cell discovery time based on the hardware specifications. In other words, we aim at spotting the user within the minimum number of attempts. To fairly evaluate DGS and EDP schemes, we introduce

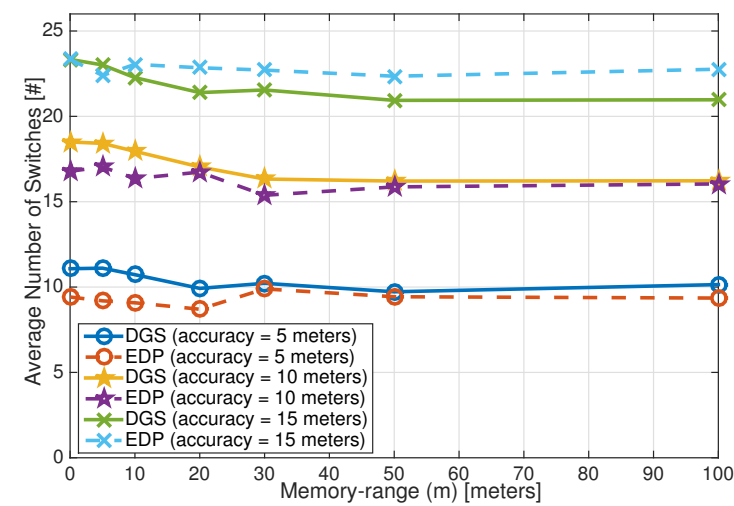

Fig. 3: Average number of discovery attempts (switches) in an obstacle-free network scenario where DGS and EDP are implemented.

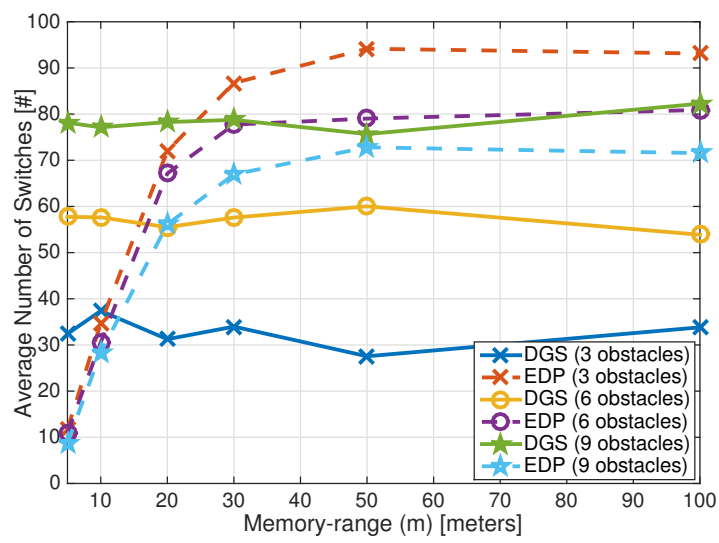

Fig. 4: Average number of discovery attempts (switches) where DGS and EDP are provided with perfect context information.

the learning approach in the greedy search, using the stored successful beamforming configurations as starting point when a newcomer announces a position within $m$ meters of distance from the stored location. For $m=0$, no additional stored information are used during the cell discovery phase.

Fig 3 shows a comparison between DGS and EDP when the memory-store feature is applied and no obstacle is placed in the area. When no learning approach is applied (e.g., memoryrange $m=0$ ), EDP outperforms the DGS approach whenever it gets a sufficient level of accuracy of user locations. When the position information error becomes consistent, EDP performs worse than DGS, as much more attempts are wasted in wrong discovery sectors. Although the memory-store approach does not bring significant gains for obstacle-less scenarios, it does not degrade the cell discovery performance. In particular, when the uncertainty in the position information is dangerously high, both approached rely on wrong stored information leading the system to work on inefficient working points. Learning approach should be activate when the location accuracy is below 10 meters (reasonable values for outdoor GPS) while promptly switched off when position information are mostly affected by errors.

\section{B. Learning approach for handling obstruction issues}

In the next evaluation set, we randomly drop in our baseline scenario opaque objects which act as obstacles within mm- 


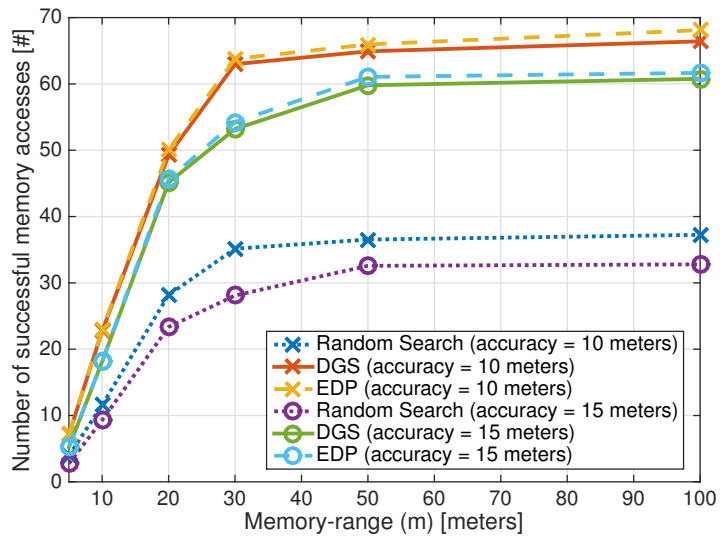

Fig. 5: Number of successful memory accesses when 9 obstacles are placed.

waves transmission paths. The obstacles are built as square of $20 \times 20$ meters size. Memory-store approach is evaluated when perfect context information are provided through the conventional cellular networks. Whenever an incoming user wants to associate with a mm-waves small cell, the cell discovery algorithm computes the correct beamforming configuration and beam the user. If a signal obstruction occurs, the search discovery algorithm needs to exploit ray reflections to find alternative directions for user covering. In Fig. 4 the average rendezvous time is shown as function of memoryrange $(m)$ parameter, when the number of obstacles placed in the area varies ${ }^{2}$. Clearly, when the number of obstacles is low (e.g., 3 obstacles), the announced user location can be likely reached through a direct beam (left points on the graph). When obstacles obstruct the transmission paths (for instance with 9 obstacles), EDP exhibits outstanding results with respect to DGS (average number of switches is 8 times less). Conversely, increasing the memory-range $(m)$ may result in a disruptive effect, when a few number of obstacles are placed. Finally, EDP still shows better results than DGS when several obstacles are placed in the network area, even though large memory-range is applied.

This is largely confirmed in Fig. 5, where a number of successful memory accesses is represented as function of memory-range $(m)$. The more memory-range distance considered, the more successful accesses performed. In addition, we also compare a random search discovery algorithm, where beamforning configurations are randomly chosen and no configuration repetition is allowed. EDP shows the maximum percentage of valid stored information which benefit the algorithm in the discovery time minimisation.

\section{Conclusions}

The most promising solution for $5 \mathrm{G}$ future network design seems to be the adoption of mm-waves technologies, which may co-exist with conventional cellular network. In particular, we have analysed the cell discovery procedure when mmwaves technologies are available and incoming users want to boost its cellular network performance. We have proposed an

\footnotetext{
${ }^{2}$ Obstacles are dropped in different places every simulation instance.
}

enhanced mechanism (EDP) which jointly exploits the contextbased information and the spatial diversity of $\mathrm{mm}$-waves antennas. In addition, we have introduced a learning approach which provides mm-waves small cell with a fingerprint map, where successful beamforming configurations are stored to speed up the discovery process upon a new user comes close to a known position. The EDP shows outstanding results in terms of discovery time providing an appealing alternative to random or greedy search algorithms.

\section{ACKNOWLEDGMENTS}

The research leading to these results has been partially supported by the EU 7th Framework Program (FP7-ICT-2013EU-Japan) under grant agreement number 608637 (MiWEBA).

\section{REFERENCES}

[1] S. Rangan, T. Rappaport, and E. Erkip, "Millimeter-wave cellular wireless networks: Potentials and challenges," Proceedings of the IEEE, vol. 102, no. 3, pp. 366-385, March 2014.

[2] A. Maltsev, A. Pudeyev, I. Karls, I. Bolotin, G. Morozov, R. Weiler, M. Peter, and W. Keusgen, "Quasi-deterministic approach to mmwave channel modeling in a non-stationary environment," in Workshop on Emerging Technologies for $5 G$ Wireless Cellular Networks, 2014.

[3] Q. C. Li, H. Niu, G. Wu, and R. Q. Hu, "Anchor-booster based heterogeneous networks with mmwave capable booster cells," in Globecom Workshops (GC Wkshps), 2013 IEEE, pp. 93-98.

[4] A. Capone, I. Filippini, and V. Sciancalepore, "Context information for fast cell discovery in mm-wave $5 \mathrm{~g}$ networks," in the 21th European Wireless Conference (EW), May 2015.

[5] "Heterogeneous Wireless Network with mmWave Small Cell Access and Backhauling," MiWaves White Paper, 2015. [Online]. Available: http://www.miwaves.eu/MiWaveS-WhitePaper January2015.pdf

[6] K. Sakaguchi, G. K. Tran, H. Shimodaira, S. Nanba, T. Sakurai, K. Takinami, I. Siaud, E. C. Strinati, A. Capone, I. Karls, R. Arefi, and T. Haustein, "Millimeter-wave evolution for 5G cellular networks," IEICE Trans. on Comm., vol. E98-B, no. 3, March 2015.

[7] R. Weiler, W. Keusgen, I. Filippini, and A. Capone, "Split control plane functionality in millimeter-wave overlay access," in 1st International Conference on $5 G$ for Ubiquitous Connectivity, May 2014.

[8] Q. Chen, J. Tang, D. Wong, X. Peng, and Y. Zhang, "Directional cooperative mac protocol design and performance analysis for ieee 802.11ad wlans," Vehicular Technology, IEEE Transactions on, vol. 62, no. 6, pp. 2667-2677, July 2013.

[9] K. Chandra, R. Prasad, I. Niemegeers, and A. Biswas, "Adaptive beamwidth selection for contention based access periods in millimeter wave wlans," in Consumer Communications and Networking Conference (CCNC), 2014 IEEE 11th, Jan 2014, pp. 458-464.

[10] G. Jakllari, W. Luo, and S. V. Krishnamurthy, "An integrated neighbor discovery and mac protocol for ad hoc networks using directional antennas," Wireless Communications, IEEE Transactions on, vol. 6, no. 3, pp. 1114-1024, 2007.

[11] T. Korakis, G. Jakllari, and L. Tassiulas, "Cdr-mac: A protocol for full exploitation of directional antennas in ad hoc wireless networks," Mobile Computing, IEEE Transactions on, vol. 7, no. 2, pp. 145-155, Feb 2008.

[12] R. Choudhury and N. Vaidya, "Deafness: a mac problem in ad hoc networks when using directional antennas," in Network Protocols, 2004. ICNP 2004. Proceedings of the 12th IEEE International Conference on, Oct 2004, pp. 283-292.

[13] C. N. Barati, S. Amir Hosseini, S. Rangan, P. Liu, T. Korakis, S. S. Panwar, and T. S. Rappaport, "Directional Cell Discovery in Millimeter Wave Cellular Networks," ArXiv e-prints, Apr. 2014.

[14] V. Sciancalepore, I. Filippini, V. Mancuso, A. Capone, and A. Banchs, "Semi-distributed mechanism for inter-cell interference coordination exploiting the absf paradigm," in the 12th IEEE International conference on SEnsing, COmmunication and Networking (SECON), May 2015.

[15] "FP7-ICT-608637 MiWEBA Project Deliverable D5.1 - Channel Modeling and Characterization," June 2014. [Online]. Available: http://www. miweba.eu/wp-content/uploads/2014/07/MiWEBA_D5.1_v1.01.pdf 